\title{
Humanistische Transpositionen
}

\author{
Die ersten deutschen Übersetzungen von Andrea Alciatos \\ Emblembuch
}

Dass mit der editio princeps des Emblematum liber 1531 eine Gattung geschaffen wurde, die Literatur und Kunst in den kommenden 200 Jahren entscheidend prägen sollte, war zu diesem Zeitpunkt nicht abzusehen. ${ }^{1}$ Das in Augsburg bei Heinrich Steiner am 28. Februar veröffentlichte Büchlein war eine Koproduktion: Veranlasst wurde die Ausgabe von Konrad Peutinger, bei den aus der Feder des Mailänder Juristen Andrea Alciato stammenden lateinischen Epigrammen handelt es sich zu einem nicht unwesentlichen Teil um Übersetzungen antiker griechischer Gedichte, die verwendeten Holzschnitte wiederum entstanden nach Zeichnungen des Augsburger Künstlers Jörg Breu des Älteren, hergestellt wurden sie wahrscheinlich von Hans Schäufelin, dessen Monogramm sich in der zeitnah erfolgten zweiten Ausgabe vom 6. April findet. ${ }^{2}$

Mit seinen Epigrammen reihte sich Alciato ein in die Übersetzungstätigkeit anderer Humanisten, die Verstexte aus der Anthologia Planudea latinisierten. ${ }^{3}$

1 Das Titelblatt der Erstausgabe kündigt den Inhalt des Drucks wie folgt an: Viri Clarissimi D. Andreae Alciati Iuris consultissimi Mediolanensis ad D. Chonradum Peutingerum Augustanum, Iurisconsultum Emblematum liber. M.D. XXXI. - Alciatos Emblembuch erlebte in den folgenden Dekaden unzählige Neuauflagen, Erweiterungen und Übersetzungen (eine große Anzahl der zwischen 1531 und 1621 edierten Ausgaben sind im Rahmen des Projekts Alciato at Glasgow digitalisiert sowie transkribiert worden; sie finden sich unter: www.emblems.arts.gla.ac.uk/alciato/ index.php [19.4.2017]).

2 Siehe zur Entstehungsgeschichte der Erstausgabe: Andreas Bässler: Die Umkehrung der Ekphrasis. Zur Entstehung von Alciatos Emblematum liber (1531). Würzburg 2012, v. a. S. 9-29; Johannes Köhler: Warum erschien der Emblematum liber von Andreas Alciat 1531 in Augsburg? In: The European Emblem. Selected Papers from the Glasgow Conference. 11-14 August 1987. Hg. von Bernard F. Scholz, Michael Bath, David Weston. Leiden 1990 (Symbola et Emblemata 2), S. 19-31; Bernhard Scholz: The Augsburg Edition of Alciato's Emblemata: A Survey of Research. In: Emblematica 5 (1990), S. 213-254.

3 Die Anthologia Planudea wurde 1494 in Florenz bei Laskaris erstmals gedruckt, 1503 folgte die Ausgabe von Aldus Manutius in Venedig. Das Epigramm war bereits aufgrund der lateinischen Vorbilder bei den Humanisten als Gattung beliebt, die Rezeption der Anthologia Planudea beflügelte die betreffende dichterische Tätigkeit in den folgenden Jahrzehnten umso mehr. Den Einfluss der Anthologia Planudea auf das 16. Jahrhundert dokumentieren: Susanna De Beer, Karl A. E. Enenkel, David Rijser (Hg.): The Neo-Latin Epigram. A Learned and Witty Genre. Leuven 2009 (Supplementa Humanistica Lovaniensia 25); Jeanne Dion: L'épigramme. De l'antiquité au 
Der Emblematum liber kann also in gewissem Sinne selbst schon als Übersetzungsliteratur betrachtet werden, wobei die Übertragung von einer Vorlage ohne abendländisch-mittelalterliche Tradition ausging, da diese erst im Zuge der vollständigen Zerstörung des oströmischen Reichs im 15. Jahrhundert nach Italien gelangt war - zusammen mit unzähligen anderen griechischen Manuskripten, die von byzantinischen Gelehrten in den sicheren Westen geschafft wurden. Der Emblematum liber ist mithin Teil des großen Projekts der Aneignung und Durchdringung der griechischen Antike, wie sie von Humanisten in ganz Europa im Lauf des 16. Jahrhunderts mit steigendem Interesse betrieben wurde.

Richtungsweisend für das graphische Layout der neuen Gattung wurde die bei Christian Wechel ${ }^{4}$ in Paris 1534 unter dem Titel Emblematum libellus veröffentlichte Version des Werks: ${ }^{5}$ Hier findet sich erstmals die typische Inanspruchnahme einer Blattseite pro Emblem, die die dreigliedrige Struktur der Text-BildKomposition deutlich macht, bestehend aus Motto, Pictura und Subscriptio. ${ }^{6}$ Wechel veranstaltete in den folgenden Jahren diverse weitere Ausgaben und brachte bereits 1536 die erste Übertragung des Emblembuchs in eine Volkssprache heraus, als zweisprachige Ausgabe konzipiert mit französischen Überset-

XVIIe siècle ou Du ciseau à la pointe. Nancy 2002 (Etudes anciennes 25); Peter Hess: Epigramm. Stuttgart 1989 (Sammlung Metzler 248); Pierre Laurens: L'abeille dans l'ambre. Célébration de l'épigramme de l'époque alexandrine à la fin de la Renaissance. Paris 1989 (Collection d'études anciennes [Série grecque] 59); Harry C. Schnur, Rainer Kößling: Galle und Honig. Humanistenepigramme. Leipzig 1982; James Hutton: The Greek Anthology in France and in the Latin Writers of the Netherlands to the Year 1800. Ithaca 1946 (Cornell Studies in Classical Philology 28); James Hutton: The Greek Anthology in Italy to the Year 1800. Ithaca 1935 (Cornell Studies in English 23); Max Rubensohn (Hg.): Griechische Epigramme und andere kleinere Dichtungen in deutschen Übersetzungen des 16. und 17. Jahrhunderts. Weimar 1897.

4 Der möglicherweise aus Basel eingewanderte Christian Wechel arbeitete seit 1522 als Buchhändler in Paris, wobei der früheste Druck, den er namentlich zeichnete, von 1526 stammt. Vgl. Hubert Elie: Chrétien Wechel. Imprimeur à Paris. In: Gutenberg-Jahrbuch (1954), S. 181-197, hier S. 182.

5 Die erste Pariser Edition präsentiert auf dem Titelblatt folgende Informationen: Andreae Alciati Emblematum Libellus. Parisiis, Excudebat Christianus Wechelus, sub scuto Basileiensi, in vico Iacobaeo. Anno M.D. XXXIIII.

$6 \mathrm{Zu}$ Wechels Ausgaben von Alciatos Emblembuch: Stephen Rawles: Layout, Typography and Chronology in Chrétien Wechel's Editions of Alciato. In: An Interregnum of the Sign: The Emblematic Age in France. Essays in Honour of Daniel S. Russell. Hg. von David Graham. Glasgow 2001 (Glasgow Emblem Studies 6), S. 49-71. Zur dreiteiligen Struktur, wie sie sich schon vor Alciatos Emblematum liber im frühen Buchdruck findet, siehe: Seraina Plotke: Emblematik vor der Emblematik? - Der frühe Buchdruck als Experimentierfeld der Text-Bild-Beziehungen. In: Zeitschrift für deutsche Philologie 129 (2010), S. 127-142. 
zungen von Jean Le Fèvre. ${ }^{7}$ Auch diese Edition folgt einem klaren graphischen Prinzip, indem jedem Emblem eine Doppelseite gewidmet ist, wobei links die Pictura zusammen mit den lateinischen Textelementen, rechts Motto und Epigramm im französischen Wortlaut präsentiert werden.

Vom Gedankengang her bleiben die französischen Textversionen nah am Sinn der jeweiligen lateinischen Vorlagen, formal jedoch besteht die Besonderheit der Transpositionen darin, dass die im elegischen Distichon gehaltenen, unterschiedlich umfangreichen lateinischen Subscriptiones fast durchwegs in Gedichte umgegossen sind, die aus acht Achtsilblern bestehen und das Reimschema abab bcbc aufweisen. ${ }^{8}$ Um diese fixe Struktur einzuhalten, hat Le Fèvre die Verszahl gegenüber dem Ausgangsepigramm öfters deutlich gekürzt, bisweilen auch erweitert. Unter den über 100 Emblemen gibt es nur wenige Ausnahmen von dieser Regel: Bei ihnen umfassen die französischen Gedichte mehr als acht Verse und weichen vom standardisierten Reimschema ab. ${ }^{9}$

\section{Wolfgang Hungers Übersetzung (Paris: Christian Wechel 1542)}

In Anlehnung an die französische Ausgabe und in analoger Gestaltung edierte Wechel 1542 die erste zweisprachige Auflage mit deutschen Übersetzungen. ${ }^{10}$ Die

7 Der Titel der ersten zweisprachigen Ausgabe lautet (inkl. auf dem Titelblatt abgedrucktem Kolophon): Livret des Emblemes, de maistre Andre Alciat, mis en rime francoyse, \& presente a mon seigneur Ladmiral de France. On les vend a Paris, en la maison de Chrestien Wechel, demeurant en la rue sainct Jaques, a lescu de Basle. M.D. XXXVI.

8 Siehe dazu: Alison Saunders: Sixteenth-Century French Translations of Alciati's Emblemata. In: French Studies 44 (1990), S. 271-288; Alison Adams: The Translator's Role in SixteenthCentury Editions of Alciati. In: Bibliothèque d'Humanisme et Renaissance 52 (1990), S. 369-383. Des Weiteren: Alison Saunders: The Sixteenth-Century French Emblem Book. A Decorative and Useful Genre. Genf 1988 (Travaux d'humanisme et Renaissance 224), S. 98-139. Zur poetischen Form des quadratischen Achtsilblers mit dem Reimschema abab bcbc siehe etwa: Jean-Michel Gouvard: La versification. Paris 1999, S. 217.

9 Dies gilt für die Embleme mit folgenden lateinischen Motti: In occasionem (Alciato, übers. von Le Fèvre [Anm.7], fol.C5v-C6r); Mutuum auxilium (fol.D3v-D4r); Submovendam ignorantiam (fol. G3v-G4r); In statuam Bacchi (fol. J8v-K2r); In simulachrum Spei (fol. L4v-L6r); $\alpha \dot{v} \varepsilon \dot{\rho} \rho \omega \varsigma$, id est, amor virtutis (fol. L8v-M1r); Eloquentia fortitudine praestantior (fol. N4v-N5r); In statuam Amoris (fol. N8v-03r). Schemagemäß, aber mit zwei französischen Epigramm-Versionen: In faci-

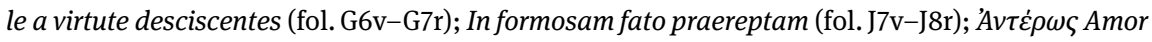
virtutis alium Cupidinem superans (fol. K6v-K7r).

10 Die Titelseite des Drucks kündigt den Inhalt wie folgt an: Clarissimi Viri D. Andreae Alciati Emblematum libellus, vigilanter recognitus, \& iam recens per Wolphgangum Hungerum Bava- 
von Wolfgang Hunger, einem bayrischen Juristen, ${ }^{11}$ hergestellten Übertragungen folgen in Umfang, Metrum und Reimschema den französischen Epigrammen, wobei die betreffende feste Struktur in der Regel auch bei denjenigen Emblemen eingehalten ist, welche in der französischen Version vom formalen Schema abweichen. ${ }^{12}$ Nur bei einzelnen besonders langen Gedichten hat sich Hunger wiederum insofern an Le Fèvre orientiert, als er ebenfalls die quadratische Form des Acht mal Acht verlässt, ${ }^{13}$ sich vom Umfang her nach dem lateinischen Original richtet und mitunter explizit vermerkt: In zal der ver $\beta$ dem Latein gleich ${ }^{14}$ oder In zal der rheymen nach dem Latein, vers gegen vers. ${ }^{15}$

Was den Sinngehalt der Text-Bild-Konfigurationen angeht, sucht Hunger bei seinen Übertragungen große Nähe zur jeweiligen Vorlage. Mitunter übersetzt er geradezu wörtlich - dies gilt vor allem auch für die Motti -, öfters nimmt er jedoch syntaktische Umstellungen vor oder ändert den Argumentationsgang zugunsten einer Zuspitzung des Gedankens, ${ }^{16}$ setzt allerdings nicht dieselben Akzente wie der französische Übersetzer. Insbesondere bei den längeren Epigrammen Alcia-

rum, rhythmis Germanicis versus. Parisiis. Apud Christianum Wechelum, sub scuto Basiliensi, in vico Iacobeo \& sub Pegaso, in vico Bellovacensi. Anno. M.D. XLII. - Diese Ausgabe ist im Nachdruck erschienen: Andreas Alciatus: Emblematum libellus. Mit einer Einleitung von August Buck. Reprographischer Nachdruck der Originalausgabe Paris 1542. Darmstadt 1991. Nachweise werden im Folgenden nach dieser Ausgabe in runden Klammern im Text gegeben.

11 Wolfgang Hunger ist 1511 in Kolping bei Wasserburg am Inn geboren, studierte zunächst an der Universität Ingolstadt, dann an der Universität Freiburg bei Ulrich Zasius. Nach weiteren Lehrjahren in Frankreich mit Studien an der für ihre juristische Fakultät renommierten Universität Bourges wurde er 1540 an die Universität Ingolstadt berufen, wo er wenig später als Rektor wirkte. Weitere berufliche Stationen waren das Amt des Assessors des Kammergerichts zu Speyer sowie des Kanzlers des Bischofs von Freising. Nur 44-jährig verstarb er 1555 während des Reichstags zu Augsburg, wo er als Gesandter anwesend war. Siehe zu Hungers Biographie und zu seinen weiteren Schriften: Johannes Köhler: Hunger, Wolfgang. In: Frühe Neuzeit in Deutschland 1520-1620. Verfasserlexikon 3 (2014), Sp. 436-440; Max Rubensohn: Einleitung. In: ders. (Hg.) (Anm.3), S. I-CCLXXVI, hier S. II-XLVI; Georg Westermayer: Hunger, Wolfgang. In: Allgemeine Deutsche Biographie 13 (1881), S. $414 \mathrm{f}$.

12 Details zur Umsetzung von Versmaß und Reim bei Hunger analysiert Rubensohn (Anm. 11), S. LXXXVI-CXXIV.

13 Dies gilt ohne expliziten Vermerk für die Embleme mit den lateinischen Motti: In occasionem (Alciato, übers. von Hunger [Anm. 10], S. 48 f.); In simulachrum Spei (S. 174-177); $\alpha v \tau \varepsilon \dot{\rho} \rho \varsigma$, id est, amor virtutis (S.182f.); Eloquentia fortitudine praestantior (S. 206 f.).

14 So formuliert ebd., S. 151 beim Emblem mit dem lateinischen Motto In statuam Bacchi (S. 150153).

15 So formuliert ebd., S. 216 beim Emblem mit dem lateinischen Motto In statuam Amoris (S. 214-217).

16 Für Beispiele siehe Peter M. Daly: The Intertextuality of Word and Image in Wolfgang Hunger's German Translation of Alciato's Emblematum liber. In: Intertextuality. German Literature and the 
tos ist der bayrische Jurist zu inhaltlichen Kürzungen gezwungen, um die vorgegebene formale Struktur einhalten zu können. In der Diktion ist Hunger prägnant und knapp, versucht auf engem Raum mit wenig Worten die Kernaussage der Vorlage zu erfassen. ${ }^{17}$ Meistens übernimmt er die Bezüge auf antike Gestalten der Mythologie oder der Geschichte, setzt bei seinen Lesern die betreffenden Kenntnisse also grundsätzlich voraus. Mitunter ersetzt er jedoch abgelegenere Namensformen, etwa in der Art von Patronymika oder anderen Umschreibungen, durch die allgemein geläufige. ${ }^{18}$

Besonders aufschlussreich für die Einschätzung des Selbstverständnisses von Hunger als Übersetzer sowie für die Frage der intendierten Adressaten sind die Paratexte der Edition. Im Unterschied zur ersten synoptischen Ausgabe von 1536, deren Titelblatt vollends in französischer Sprache gehalten ist und die den Text-Bild-Kompositionen sowohl ein lateinisches als auch ein französisches Widmungsschreiben voranstellt, werden die lateinisch-deutschen Embleme im Druck von 1542 ausschließlich durch lateinische paratextuelle Rahmungen eingeführt. Evidentermaßen war die Veröffentlichung nicht für ein Publikum bestimmt, das über keine Lateinkenntnisse verfügte. Während die Edition mit Le Fèvres Übertragungen im Titel die Spezifik der Transposition in gereimte französische Verse hervorhebt, den Umstand hingegen, dass die lateinischen Originale Alciatos mit abgedruckt sind, unerwähnt lässt, ${ }^{19}$ erfährt man auf dem Titelblatt der Publikation mit Hungers Gedichten lediglich in lateinischer Sprache, dass der Druck deutsche Textpassagen bereitstellt: ${ }^{20}$ Rekurriert wird im Wortlaut des Titels zunächst auf die Überschrift der Pariser Erstausgabe, sodann ist die Übersetzungleistung Hungers charakterisiert mittels der Formulierung rhythmis Germanicis versus. Dass sich hinter der schlichten Bezeichnung rhythmi Germanici das originelle Experiment verbirgt, auf deutsch die französischen quadratischen Achtsilbler mit dem Reimschema der Vorgängerübertragung nachzuahmen, erschließt sich über diese Angabe in keiner Weise.

Die Ankündigung auf der Titelseite richtet sich offenkundig an eine humanistisch gebildete Leserschaft. Es ist davon auszugehen, dass der deutschstämmige Verleger Wechel Rezipienten im Blick hatte, welche die geistige Herkunft

Visual Arts. Hg. von Ingeborg Hoesterey, Ulrich Weisstein. Columbia S.C. 1993 (Studies in German Literature, Linguistics, and Culture), S. 30-46.

17 Ausführlich mit den stilistischen Aspekten von Hungers Übersetzungen beschäftigt sich Rubensohn (Anm. 11), S. CXXIV-CL.

18 Siehe dazu Daly: Intertextuality (Anm. 16), S. 33.

19 Siehe Anm. 7.

20 Vgl. dazu auch Ingrid Höpel: Emblem und Sinnbild. Vom Kunstbuch zum Erbauungsbuch, Frankfurt/M. 1987, S. 58. 
der Embleme Alciatos einzuordnen wussten und sich auch darüber im Klaren waren, dass mit der Übertragung Hungers nicht zuletzt insofern Neuland betreten wurde, als sie den Versuch wagte, die ursprünglich griechischen Gedichte auf deutsch in eine Form zu gießen, die ihrer illustren Herkunft gerecht werden sollte, indem ein Versmaß gewählt wurde, das sich in der französischen Sprache für die Gattung des Epigramms bewährte. ${ }^{21}$ Dass es für ein derartiges Unterfangen keine Vorbilder gab, da Deutsch weit davon entfernt war, als Sprache des Humanismus etabliert zu sein, ${ }^{22}$ war für Wechel offensichtlich gerade Argument, die Ausgabe zu realisieren, obwohl abzusehen war, dass sie sich in Frankreich nicht als Verkaufsschlager durchsetzen würde. ${ }^{23}$

$\mathrm{Zu}$ diesem Konzept passt, dass auch die Vorreden zur Ausgabe mit den deutschen Übertragungen nur in Latein gegeben werden. Die kurze Anrede des Druckers an den Leser hat apologetischen Charakter, indem Wechel erklärt, dass er aus graphischen Gründen die in Hungers Widmungsschreiben angekündigte Dreisprachigkeit der Ausgabe nicht habe umsetzen können (S.2). Er verweist zudem auf die Disparatheit der Interessen, die dazu führe, dass nicht dieselben Personen an deutschen und an französischen Gedichten Gefallen fänden; Hungers Anliegen des Sprachenvergleichs nimmt er jedoch insofern auf, als er zuletzt auf die erneute Auflage der französischen Edition aufmerksam macht und erklärt, der intendierte Vergleich könne bei Bedarf unter Beizug der beiden Veröffentlichungen von 1542 realisiert werden (ebd.).

Hungers ausführliches Widmungsschreiben wird ebenfalls nur in lateinischer Sprache geboten und richtet sich an die Brüder Balthasar und Werner Seybolsdorf (S.3). Die sich über mehr als zehn Druckseiten erstreckende Vorrede gibt Einblick in die Übersetzungsmotivationen und äußert sich zum Zweck der Ausgabe. ${ }^{24}$ Sie ist auf den 1. Mai 1539 datiert, als Unterzeichnungsort ist Bourges

$21 \mathrm{Zu}$ den Anfängen der französischen Epigrammatik siehe Friedrich Fuchs: Beitrag zur Geschichte des französischen Epigramms 1520-1800. In: Das Epigramm. Zur Geschichte einer inschriftlichen und literarischen Gattung. Hg. von Gerhard Pfohl. Darmstadt 1969, S. 235-283, hier S. 235-252.

22 So bezeichnet Max Rubensohn - mit Verweis auf Ernst Höpfner - Hunger als „den ersten [...] unter den Männern, die durch Nachbildung der französischen sylbenart eine Regulierung des deutschen Verses erstrebten und dadurch gleichfalls Vorläufer Martin Opitzens wurden“, Rubensohn (Anm. 11), S. CII.

23 Im Unterschied zur französischen Übersetzung, welche 1542 schon die dritte Auflage erlebte, wurde die Edition mit Hungers Übertragungen denn auch nur einmal gedruckt.

24 Eine englische Übersetzung der Vorrede Hungers bietet: Denis L. Drysdall: Defence and Illustration of the German Language: Wolfgang Hunger's Preface to Alciati's Emblems (Text and Translations). In: Emblematica. An Interdisciplinary Journal for Emblem Studies 3 (1988), S. $137-$ 160. 
genannt (S. 13). Wie aus dem Auftakt des Schreibens hervorgeht, hatten sich die beiden adligen Adressaten zu einem früheren Zeitpunkt in Frankreich zu Ausbildungszwecken in Hungers Obhut befunden, hatte er sie zwei Jahre zuvor in die Heimat begleitet und dort nach einer neuen Beschäftigung gesucht, war dann jedoch aus hier nicht weiter ausgeführten Gründen nach Frankreich zurückgekehrt und stand von da an in brieflichem Kontakt mit den Schützlingen, um ihnen Neuigkeiten aus der Ferne zu berichten (S. 3 f.). Als frappierende Nachricht verkündet Hunger den beiden, er habe Alciatos Emblembuch übersetzt - Andreae Alciati Emblemata, rhythmis in linguam nostram a me versa (S.3) -, was, wie er unterstellt, die Widmungsträger sicherlich in Erstaunen versetze, weil er damit vom Juristen zum deutschen Dichter - Poeta Germanicus (ebd.) - geworden sei, und dies erst noch in Frankreich.

Signifikant ist bei dieser Thematisierung der Übersetzungstätigkeit der Umstand, dass Hunger die eigenen Gedichte als Übertragungen der Epigramme Alciatos betrachtet, obgleich er sich bei seinem Unterfangen wesentlich an den französischen Versen Le Fèvres orientierte. Die betreffenden Bemühungen machen ihn in seinen Augen zum Poeta Germanicus, eine Formulierung, die sowohl die dichterische Eigenleistung unterstreicht - die als Teil des Übertragungsvorgangs gewertet wird - als auch die Einschätzung der poetischen Neuschöpfung als humanistisch geprägtes Produkt hervorhebt. Wenn auch die entsprechende Selbstbezeichnung hier durchaus mit einem Augenzwinkern den befreundeten Adressaten gegenüber erfolgt, ${ }^{25}$ macht Hunger doch deutlich, dass die Kategorie des deutschen - im Sinne des deutschsprachigen - Dichters, der sich den klassischen Sprachen und deren Literatur verpflichtet, konzeptuell zum hier präsentierten poetologischen Programm gehört. Dass Hunger seine Wandlung vom Juristen zum Dichter in dieser Weise betont, lässt sich darüber hinaus als Hinweis darauf lesen, dass er sich mit seiner Tätigkeit gewissermaßen in die Nachfolge Alciatos stellt, der - ebenfalls Jurist - einige Jahre zuvor in Bourges als Professor gewirkt hatte. ${ }^{26}$

Im weiteren Verlauf des Widmungsschreibens führt Hunger aus, dass er Alciatos Epigramme ursprünglich nur für sich selbst übersetzt habe, um sich im Gebrauch der deutschen Sprache zu üben: Als Motivation der Übertragung nennt er zunächst die Verfeinerung seiner eigenen Sprachfertigkeiten nach einem län-

25 Hunger pointiert zugespitzt (Alciato, übers. von Hunger [Anm. 10], S. 3): An non mirifica haec est metamorphosis, ut repente ex Iureconsulto vobis Poeta Germanicus prodirem?

26 Hunger dürfte Alciato allerdings nicht persönlich in Bourges gehört haben, Alciato war von 1529 bis 1533 dort tätig (vgl. Giuseppe Cuscito: Andrea Alciato. In: Personenlexikon zur christlichen Archäologie 1 [2012], S. 58 f.). 
geren Frankreichaufenthalt - gerade im Hinblick auf die verbale Gewandtheit, wie sie vor Gericht von Nutzen sei -, charakterisiert dabei die Konkurrenz mit den heimischen Anwälten und Rechtsbeamten explizit als von Missgunst geprägte Rivalität (S.4). Hunger spricht in diesem Zusammenhang von mihi acquirendam copiam, cultumque lautiorem linguae nostratis Germanicae (ebd.), verdeutlicht damit im Umkehrschluss aber gerade auch, dass die Übersetzungen Fülle und Raffinesse der deutschen Sprache seiner Meinung nach zum Ausdruck zu bringen in der Lage sind.

Der Jurist markiert seine deutschen Verse mit diesen Überlegungen einerseits als eine Art Übungsfeld, auf welchem in spielerischer Weise für den konkreten Ernst der verbalen Auseinandersetzung im ,richtigen“ Leben Vorbereitungen getroffen werden können, andererseits wird schon hier das poetologische Programm greifbar, die Epigramme als Evidenz der Möglichkeiten dichterischer Praxis in deutscher Sprache zu bestimmen. Der Übungscharakter, den Hunger seinen Gedichten zuschreibt, korrespondiert zudem mit der literarischen Gepflogenheit seiner humanistischen Zeitgenossen, die eigenen poetischen Erzeugnisse als Progymnasmata zu bezeichnen, zumal in Zusammenhängen, wo dichterisches Neuland betreten wird. ${ }^{27}$

Der Gesichtspunkt des Spielerischen, den Hunger mit seiner poetischen Übersetzungstätigkeit verbindet, kongruiert mit dem ,Sitz im Leben“, den er Alciatos Emblemen zumisst: Diese dienen ihm nämlich, wie er konstatiert, zur Abwechslung und zum Vergnügen während des Frühstücks oder Mittagessens - und zwar in der zweisprachigen Ausgabe mit den Epigrammen Le Fèvres (ebd.). Den Ausschlag, gerade dieses Büchlein als Ausgangspunkt für seine Übertragungen zu wählen, gab für Hunger denn nicht nur der Umstand, dass ihm Alciatos Embleme

27 So hat beispielsweise Thomas Morus in die Erstausgabe seiner gesammelten Epigramme zum Auftakt unter dem Titel Progymnasmata achtzehn griechische Gedichte sowie deren von ihm selbst und von seinem Freund William Lily fabrizierten lateinischen Übersetzungen aufgenommen. Die Mehrzahl der vornehmlich aus der Anthologia Planudea stammenden Epigramme hat von beiden Humanisten je eine Übersetzung erhalten, bei zwei Gedichten sind neben Lilys Version sogar zwei Varianten von Morus abgedruckt. Wie der Titel verdeutlicht, handelt es sich bei diesen Übersetzungen gleichsam um Fingerübungen, um im spielerischen Wettstreit entstandene Übertragungen griechischer Epigramme ins Lateinische (in: De optimo reip. statu, deque nova insula Utopia, libellus vere aureus, nec minus salutaris quam festivus, clarissimi disertissimique viri Thomae Mori inclytae civitatis Londinensis civis et vicecomitis; Epigrammata clarissimi disertissimique viri Thomae Mori, pleraque e Graecis versa. Epigrammata Des. Erasmi Roterodami. Basel: Johann Froben 1518). Die drei Teile des Drucks sind durch separate Titelblätter abgetrennt, bei fortlaufender Paginierung, und werden durch eigene Widmungsschreiben eingeleitet; die Progymnasmata eröffnen den Teil der Epigrammata von Thomas Morus im Anschluss an die Dedikationsepistel von Beatus Rhenanus an Willibald Pirckheimer. 
besonders geeignet schienen, weil sie seiner Ansicht nach in so angenehmer Weise Nützliches und Erfreuliches verknüpfen, sondern auch, weil ihn die französischen Verse animierten, es auf Deutsch in derselben Weise zu versuchen: videoque rhythmis versum Gallice, placuit ilico eodem carminis genere tentare versionem Germanicam (ebd.). Wie Hunger hier expliziert, ist es nicht zuletzt die im Französischen verwendete künstlerische Form, die ihn herausfordert: Er ist, so führt er unmittelbar darauf aus, an der französischen Sprache als poetischer interessiert und möchte im Sinne eines dichterischen Experiments erproben, ob es gelänge, auf deutsch derartige Verse zu bilden. ${ }^{28}$

Nachdem Hunger den Aspekt der stilistischen Verfeinerung des Ausdrucks in deutscher Sprache, die sich in der Fülle verbaler Möglichkeiten und im versierten Gebrauch des Redeschmucks offenbare, noch einmal stark gemacht hat (S. 5), geht er näher auf die Umstände der Drucklegung seiner Epigramme ein. Er rechtfertigt die Transpositionen in diesem Zusammenhang mehrfach ausdrücklich als spielerische Leistung (ludens, lusus) und gibt zu bedenken, dass solche Schritte ins unbekannte Terrain immer Kritiker hervorrufen würden, es indessen jedem offenstünde, ihn mit einem eigenen Versuch zu überflügeln (S. 5-8).

Fragen zum Verhältnis von vergnüglicher Abwechslung und ernsthaftem Nutzen, kombiniert mit Überlegungen zur Relation von poetischer Übersetzungstätigkeit und rechtswissenschaftlicher Expertise bringen Hunger zu einem weiteren grundsätzlichen Argument hinsichtlich der Einschätzung seiner deutschen Gedichte. So geht er vergleichend auf die drei involvierten Sprachen ein, wobei er zunächst als Zweck seiner Übertragungen die Möglichkeit der Gegenüberstellung von Französisch und Deutsch thematisiert, indem er festhält:

Nam \& qui nostrae Gallicaeque linguae studiosi sunt, hic tanquam in speculo facili collatione contemplabuntur, huius vel illius in quibusdam ubertatem, inopiam, suavitatem, ruditatem, mollitiem aut asperitatem, idque genus consimilia. (S.9)

Gemäß Hunger können diejenigen, die sich sowohl um das Deutsche wie um das Französische bemühen, den Sprachenvergleich dahingehend am Beispiel der beiden Alciato-Übersetzungen vornehmen, als sie Fülle, Eleganz und Geschmeidigkeit im Wechsel beurteilen. Indem der Jurist als zentrale Kategorien ubertas vs. inopia, suavitas vs. ruditas und mollities vs. asperitas nennt, setzt er Maß-

$28 \mathrm{Zu}$ diesem Gedanken Ingrid Höpel (Anm. 20), S. 60: „Nicht im Hinblick auf potentielle Leser übersetzt Hunger Alciatus; ihm geht es vielmehr um die Bereicherung und Vervollkommnung der deutschen Sprache. Nachdem er festgestellt hat, dass die französische Fassung der ursprünglich lateinischen ebenbürtig ist, nimmt er sich vor, der deutschen Sprache eine ähnliche Leistung abzuverlangen.“ 
stäbe, wie sie von der klassischen Rhetorik in Anschlag gebracht worden sind. ${ }^{29}$ Dadurch, dass er selbst hinsichtlich des Ergebnisses der abwägenden Überprüfung zunächst nicht Stellung bezieht, sondern die Bilanzierung dem geneigten Leser überträgt, insinuiert er mit den angesetzten Leitlinien, dass die beiden Sprachen seiner Gegenwart das Potenzial haben, in ihrer Wertigkeit an das Latein der Vorbilder heranzukommen. Auch Deutsch und Französisch werden mit dieser Ausgangssituation vorerst als ebenbürtig betrachtet, was Hunger nicht zuletzt dadurch verdeutlicht, dass er eigens konstatiert, er habe gerade um dieses Vergleichs willen bei seinen deutschen Übersetzungen dieselbe metrische Form gewählt, wie sie die französischen Übertragungen bieten (ebd.).

Scheint es ihm bis zu diesem Punkt durchaus angelegen zu sein, das Deutsche dem Französischen gleichzustellen und damit auch eine gewisse Eigenwertigkeit gegenüber dem Lateinischen zu postulieren, macht er im Folgenden klar, dass es die beiden Volkssprachen im Bereich der Poesie seiner Meinung nach keineswegs mit den antiken Mustern aufnehmen können: Praeterea nihil obscure hic perspicitur, quam frigidi \& insuaves sint cuiusvis linguae rhythmi, ad tersissimas Musas Latinorum (ebd.). Das hier mit einiger Schärfe formulierte Verdikt bezieht Hunger aber gleich auch auf die Italiener, welche mit fehlgeleiteter Arroganz glauben würden, ihre toskanischen Gedichte wären gar in der Lage, die lateinischen Musen zu überflügeln (ebd.). Wie in dieser letzten Wendung der Argumentation augenscheinlich wird, nimmt der Übersetzer hier seine experimentierfreudige Position zurück zugunsten einer wertkonservativen Haltung, die der Sprache der Lehrmeister die klare Prävalenz einräumt. Damit geht einher, dass er in einem zweiten Schritt auch eine Hierarchie zwischen den französischen und den deutschen Epigrammen konzediert, indem er den Gedichten in seiner Muttersprache im Vergleich weniger geschliffene Eleganz zubilligt, mit der Begründung: praeter monosyllaborum asperitatem, quibus lingua Germanica plena est (ebd.).

Zwar stellt Hunger mit diesen letzteren Ausführungen seine Übertragungen ins Deutsche hinter die Epigramme der Vergleichssprachen Latein und Französisch, trotzdem markiert er zum Schluss seiner Vorrede nochmals die eingangs eingeschlagene Stoßrichtung des Gedankengangs. Er möchte sich den Rechtsvertretern seiner Heimat, die für sich selbst gewandten Sprachgebrauch reklamierten, entgegenstellen mit einer deutschen Ausdrucksform, die humanistisch geschult ist (S.9 f.). Explizit wirft er den deutschen Anwälten und Notaren vor, sie könnten weder Latein noch sonst eine Fremdsprache, weswegen er ihnen auch das Urteilsvermögen hinsichtlich der von ihm übersetzten Gedichte abspricht (S. 10). Umso mehr sieht er den Zweck der mehrsprachigen Ausgabe von Alciatos

29 Siehe etwa die ersten Kapitel des 10. Buchs von Quintilians Institutio oratoria. 
Emblembuch in der Möglichkeit, Jugendlichen die Schönheit von Tugenden und das Übel der Laster in einprägsamer Weise vor Augen zu stellen, da er die TextBild-Verbindungen unter didaktischen Gesichtspunkten - wie er unter Rückgriff auf antike Gewährsleute ausführt - als besonders wertvoll einschätzt (S. 10 f.). ${ }^{30}$

Auch wenn Hunger hier mit seiner Argumentation Überlegungen aufgreift, wie sie im Zusammenhang mit der Rezeption antiker Epigramme und insbesondere Alciatos Emblemen von seinen Zeitgenossen ebenfalls vorgebracht wurden, gibt er ihnen doch eine spezifische Wendung, indem er die Schulung an einer der humanistischen Lieblingsgattungen wie dem lateinischen Epigramm mit der Verfeinerung deutscher Sprachkompetenz zusammenbringt. So möchte er offenkundig gerade angehende Juristen in seiner Heimat dafür begeistern, dass sie am europäischen Projekt humanistischer Gelehrsamkeit insofern teilhaben, als sie das Studium zeitgenössischer Rechtsliteratur mit poetischer Praxis verbinden, wobei er dafür - gleichsam als Köder - im humanistischen Geiste entstandene deutschsprachige Gedichte nutzbar machen will:

nihil movere vos debet, iuvenes ornatiss[i]mi, quin studiorum occupationibus suffuremini ociolum aliquod, si possitis, quod libelli huius lectioni impertiatis. Tum \& caetera omnia Alciati diligenter volvite, neque unquam deponite e manibus. (S.12)

Andrea Alciato selbst wird als Paradebeispiel eines Mannes vorgestellt, der die Kombination von spielerischer Geistesbildung und juristischer Kennerschaft in vorbildlicher Weise verkörpert, wie auch in dessen rechtswissenschaftlichen Publikationen sichtbar werde, die Hunger seinen Schützlingen ebenfalls anempfiehlt. $^{31}$

Alles in allem wird bei der nicht durchgehend stringenten Argumentation des Widmungsschreibens Hungers eines deutlich: Der bayrische Jurist betrachtet seine Übersetzungen von Alciatos Emblemen vor allem als agonales Spielfeld, und zwar in verschiedener Hinsicht. Grundsätzlich geht es ihm um Wetteifern, wie es den Renaissance-Humanismus als solchen konzeptuell prägt. Auf der

30 Siehe dazu auch: Johannes Köhler: Der Emblematum liber von Andreas Alciatus (1492-1550). Eine Untersuchung zur Entstehung, Formung antiker Quellen und pädagogischen Wirkung im 16. Jahrhundert. Hildesheim 1986 (Beiträge zur historischen Bildungsforschung 3), S. 58-60.

31 Alciato gilt als zentraler Vertreter der humanistischen Jurisprudenz, des sogenannten mos gallicus, gemeinsam mit Ulrich Zasius und Guillaume Budé, die sich von den Vertretern des mos italicus absetzten, indem sie die humanistische Quellenkritik als Methode auf das Corpus Iuris Civilis übertrugen (siehe weiterführend etwa: Hans Erich Troje: Humanistische Jurisprudenz. Studien zur europäischen Rechtswissenschaft unter dem Einfluss des Humanismus. Goldbach 1993 [Bibliotheca eruditorum. Internationale Bibliothek der Wissenschaften 6]). 
Ebene der poetischen Produkte manifestiert sich in Hungers Transpositionen das Wetteifern der Sprachen, wobei als besonders bemerkenswert hervorzuheben ist, dass nicht mehr nur Italienisch oder Französisch in den Wettbewerb mit Latein und Griechisch eintreten, sondern Deutsch von Hunger als satisfaktionsfähig eingestuft wird, auch wenn es zuletzt nur den Trostpreis erhält. Die deutsche Sprache wird hier insofern nobilitiert, als sie überhaupt zur Konkurrenz zugelassen wird.

In einer zweiten Hinsicht thematisiert die Vorrede die Rivalität der deutschen Juristen untereinander, wobei Hunger die Gruppe der humanistisch Gebildeten von denjenigen unterscheidet, welche sich seiner Meinung nach in missgünstiger Weise für etwas Besseres halten, obwohl sie sich in überkommenen Bahnen bewegen. ${ }^{32}$ In diesem Zusammenhang wird sein didaktisches Anliegen virulent, indem er Auszubildenden als Grundhaltung näherzubringen sucht, dass zur fachlichen Expertise auch die Fähigkeit gehört, seinen Geist im Bereich der Poesie zu schulen, beispielsweise an Epigrammen - als derjenigen Gattung, die Abwechslung in den Alltag und in die Berufsgeschäfte bringt, zugleich Einsichten über das menschliche Wesen und dessen Charaktereigenschaften vermittelt. ${ }^{33}$ Hunger vertritt diesbezüglich den Standpunkt, dass sich selbst die muttersprachlichen Kompetenzen vor Gericht verbessern lassen, wenn der literarischen Auseinandersetzung mit derartigen Texten angemessen Zeit eingeräumt wird.

Nicht zuletzt tritt Hunger selbst in einen Agon mit den großen europäischen Humanisten, von denen einige von Beruf Juristen sind, zu deren Selbstverständnis die literarische Tätigkeit gehört, insbesondere die Auseinandersetzung mit der Gattung des Epigramms. Als Meister und Vorbild nennt er ausdrücklich Andrea Alciato, doch auch sein Freiburger Lehrer Ulrich Zasius, Konrad Peutinger oder Thomas Morus könnten hier ins Feld geführt werden. Hunger wetteifert insofern mit seinen berühmten Zeitgenossen, als er sich zutraut, dichterisches Neuland zu betreten und Wege zu gehen, die man als solche erst schlagen musste.

32 Dies lässt sich als Positionierung Hungers im juristischen Methodenstreit zwischen mos gallicus und mos italicus interpretieren. Bourges, der angegebene Unterzeichnungsort des Widmungsschreibens, war zu diesem Zeitpunkt eine Hochburg der Anhänger des mos gallicus und ist von daher sicherlich auch programmatisch gewählt.

33 Vgl. etwa Seraina Plotke: Epigrammatik im Gattungsverständnis des frühen 16. Jahrhunderts. Die Epigramme von Thomas Morus und Erasmus von Rotterdam in der Ausgabe Froben 1518. In: Le ,sel` antique: Epigramme, satire, théâtre et polémique. Das ,Salz‘ der Antike. Epigramm, Satire, Theater, Polemik. Hg. von Marie-Laure Freyburger-Galland, Henriette Harich-Schwarzbauer. Stuttgart 2016, S. 191-200. 


\section{Die Übersetzung von Jeremias Held (Frankfurt: Georg Rab für Sigmund Feyerabend und Simon Hüter 1566/1567)}

Ein in jeder Hinsicht anderes Bild zeigt sich im Druck der von Jeremias Held ${ }^{34}$ gefertigten zweiten deutschen Übersetzung von Alciatos Emblemen. Sie ist 1566/1567 erstmals erschienen, verlegt durch Sigmund Feyerabend und Simon Hüter, gedruckt bei Georg Rab in Frankfurt am Main. ${ }^{35}$ Auch diese Ausgabe ist zweisprachig gehalten, allerdings folgt sie, was die Präsentation der Embleme angeht, nicht dem von Wechel etablierten einheitlichen Druckbild, welches Motto, Pictura und Subscriptio einerseits, die übersetzten Textelemente andererseits auf einer Doppelseite unterbringt: Feyerabends Publikation reiht platzsparend sämtliche Komponenten hintereinander, wobei die Pictura - die Seitenökonomie berücksichtigend - mal im Verbund mit den lateinischen, mal mit den deutschen verbalen Bestandteilen präsentiert wird, mitunter ganz fehlt. Was den Umfang der übersetzten Epigramme betrifft, hat Held die Anzahl Verse gegen-

34 Über Jeremias Held ist wenig bekannt. Auf dem Titelblatt zur Übersetzung von Alciatos Emblembuch nennt er als Herkunftsort Nördlingen. In der Forschung geht man meistens davon aus, dass er Arzt war, im Wesentlichen aufgrund der Tatsache, dass Held auch als Verfasser eines Kräuterbuchs hervorgetreten ist. Tatsächlich war Held evangelischer Pfarrer, wie kürzlich in einer etwas abgelegeneren Publikation von Gerhard Münzing herausgearbeitet wurde. Münzing hat folgende biographischen Daten zu Held zusammengetragen: Der Vater Heinrich Held war seinerseits Pfarrer in Thann (heute Bühlertann bei Schwäbisch Hall), beteiligte sich $1525 \mathrm{am}$ Bauernkrieg und floh nach Nördlingen, wo er festgesetzt wurde; zudem war er offenbar 1535 als Pfarrer in Yppesheim (Franken?) und 1537 in Boll (am Neckar?) tätig. Der Sohn Jeremias war bis 1556 Diakon zu Achterdingen (Echterdingen bei Stuttgart) und 1556-1561 im Pfarramt zu Aistaig (gehört heute zu Oberndorf am Neckar). In den Jahren der Beschäftigung mit Alciatos Emblembuch war er Pfarrer zu Flein (bei Heilbronn), nämlich von 1565 bis 1568. Danach war er Pfarrer zu Heuchlingen (bei Heilbronn?). Held ist 1572 gestorben, als Vater von acht Kindern. Vgl. [Gerhard Münzing]: Jeremias Held. Pfarrer zu Flein. 1565-1568. Flein 2007, S. 3 f.

35 Die Titelangaben lauten: Liber Emblematum D. Andreae Alciati nunc denuo collatis exemplaribus multo castigatior quam unquam | antehac editus. Kunstbuch Andree Alciati von Meyland, bey der Rechten Doctorn, allen liebhabern der freyen Künst / auch Malern / Goldschmiden / Seidenstickern und Bildhauwern / jetzund zu sonderm nutz und gebrauch verteutscht und an tag geben / durch Jeremiam Held von Nördlingen / mit schönen, lieblichen / neuwen / kunstreichen Figuren geziert und gebessert. Mit Römischer Keyserlicher Majestät Freyheit in zehen jaren nicht nachzudrucken. Gedruckt zu Franckfurt am Mayn / M.D.LXVI. - Im Kolophon dieser Ausgabe findet sich die Datierung 1567, eine zweite Auflage erfolgte 1580. Die Erstausgabe hat folgenden Nachdruck erhalten: Jeremias Held: Liber Emblematum (Frankfurt/M. 1566). With an Introduction by Peter M. Daly. Turnhout 2007 (Imago Figurata Editions 4). 
über der lateinischen Vorlage in aller Regel genau verdoppelt, ${ }^{36}$ nicht zuletzt deshalb, um inhaltlich nah am Original zu bleiben und nicht kürzen zu müssen. Seine Gedichte sind in Knittelversen gehalten, durchgehend in der strengen Form mit acht oder neun Silben, wobei sich die distichische Struktur der lateinischen Vorlage graphisch spiegelt, indem auch auf deutsch jeweils der zweite Vers eingerückt ist. Von der Diktion her steht für Held offenkundig nicht sprachliche Prägnanz im Vordergrund, ${ }^{37}$ sondern die vergleichsweise wortgetreue Übertragung, wobei er sich vor allem dann von der Vorlage entfernt, wenn ihm Alciatos Text inhaltlich als zu voraussetzungsreich erscheint.

Bereits mit dem Titelblatt des Drucks wird augenscheinlich, dass sich die Ausgabe in erster Linie an ein Publikum richtete, das nicht humanistisch gebildet war und auch nicht über Lateinkenntnisse verfügte. Nach kurzen Informationen zur Veröffentlichung in lateinischer Sprache folgt ein selbständiger deutscher Titel, der werbewirksam den Interessentenkreis anvisiert, indem er verschiedene kunsthandwerkliche Berufsvertreter nennt - deren Auftraggeber als Käufer wohl mit in den Blick genommen werden - und die zweckmäßige Verwendbarkeit der Übersetzungen für sie betont: ${ }^{38}$ allen liebhabern der freyen Künst/ auch Malern/Goldschmiden/ Seiden-stickern und Bildhawern/ jetzund zu sonderm nutz vnd gebrauch verteutscht. ${ }^{39}$ Schon die Bezeichnung des Emblembuchs als Kunst$b_{u} h^{40}$ weckt eine Reihe von Assoziationen, wobei sowohl Arznei und Magie als auch andere Formen von Wissenschaft im weitesten Sinn anklingen. ${ }^{41}$ Indem

36 Nur beim Emblem CLXIIII (Alciato, übers. von Held (Anm.35), fol.105v-106r) bleibt die Anzahl Verse analog der lateinischen Vorlage (vgl. dazu Peter M. Daly: Introduction. In: ebd., S. 1-21, S. 14 f.).

37 Wenig schmeichelhaft ist die Charakterisierung von Helds Epigrammen, wie Max Rubensohn sie, gerade im Vergleich mit Hungers Übersetzungen, vornimmt (Rubensohn [Anm. 11], S. CLXXCLXXII): „Statt der peinlichen Sorgfalt, die Hunger nach französischem Vorbild auf die Reinhaltung des Reimes, auf wohllautende rhythmische Gliederung der einzelnen Verse verwandte, statt seiner rühmlichen Gewissenhaftigkeit auch in Hinblick auf die sprachliche Form und den Periodenbau: bei Held wieder jene Rohheit der Reimbildung, jene oft barbarische Gleichgiltigkeit gegen die einfachsten Regeln der Rhythmik, jene Vergewaltigung der Sprache ,durch Zusammenpressung und willkürliche Verdrehung der Silben', die uns selbst das, was etwa besser gelungen ist, verleiden muß. Außer der Achtsilbigkeit und dem Reim, die aber beide oft nur durch äußerst gewaltsame Mittel, oft auch nur zum Schein festgehalten sind [...], kennt Held keinerlei Schranke seiner Freiheit. Von einem prinzipiellen Festhalten am jambischen Rhythmus [...] kann bei ihm nicht die Rede sein.“

38 Vgl. Höpel (Anm. 20), S. 59.

39 Vgl. Anm. 35.

40 Vgl. ebd.

41 Dazu Höpel (Anm. 20), S. 64. 
der Nutzen des Büchleins gerade für bildkünstlerisch tätige Berufsgruppen hervorgehoben wird, schränkt der Druck den Terminus indes ein in Richtung der semantischen Verengung, wie sie sich in der Moderne vollends durchgesetzt hat. Der deutsche Titelzusatz erklärt Alciatos Emblembuch zu einem Reservoir an bildhaft gestaltbaren Motiven und deren Ausdeutungen, nimmt damit einen Anwendungsbereich der betreffenden Text-Bild-Konfigurationen vorweg, wie er vornehmlich im 17. Jahrhundert mit der buchexternen Emblematik große Relevanz erlangte. ${ }^{42}$

Wie das Titelblatt spiegeln auch die Einleitungsstücke bereits aufgrund der Sprache, in der sie gehalten sind, den intendierten Rezipientenkreis. Konträr zu Wechels Edition mit Hungers Übertragungen werden die beiden, die Ausgabe eröffnenden, Schreiben des Übersetzers nur auf Deutsch gegeben. Es handelt sich um eine knapp zehnseitige Zueignung an den Juristen Raymundus Graff sowie eine noch etwas längere Vorrede an den Günstigen Leser. Im Widmungsbrief, in welchem Held den Dedikationsträger als seinen günstigen Herrn anspricht, ${ }^{43}$ stellt er vielfältige Überlegungen zur Dankbarkeit an, die er dem Adressaten gegenüber zum Ausdruck bringen möchte. Dabei tritt er als jemand auf, der über klassische Gelehrsamkeit verfügt und diese einem weniger beschlagenen Publikum näherzubringen sucht. Er stilisiert sich zum Vermittler humanistischer Bildungsgüter an eine Klientel, der diese Welt aufgrund mangelnder Ausbildung - insbesondere in den Sprachen der Antike - nicht offensteht.

Dieser Impetus wird bereits zum Auftakt der Dedikation sichtbar, wo Held auf den Ajax von Sophokles rekurriert, den er zitiert, indem er das herangezogene Diktum zuerst in lateinischem Wortlaut formuliert und unmittelbar darauf in deutscher Übersetzung wiedergibt. ${ }^{44}$ Er macht damit gleich zu Beginn des Büchleins klar, dass ihm selbst nicht nur die lateinische, sondern auch die griechische

42 Tatsächlich hat auch Alciato selbst seine Epigramme schon früh als Vorlagen für Kunsthandwerker wie Maler oder Goldschmiede betrachtet, so in einem Brief aus dem Jahr 1523, der in der Emblemforschung immer wieder im Zusammenhang mit der Gattungsbezeichnung Emblemata zitiert wird. Vgl. Gian Luigi Barni (Hg.): Le lettere di Andrea Alciato, giureconsulto. Florenz 1953, S. 46.

43 Die Widmung lautet (Alciato, übers. von Held [Anm. 35], fol. A2r): Dem Ehrnvesten/ Hochgelehrten/ Ehrnwirdigen und Wolweisen Raymundo Graff/ der Rechten Doctorn/ Nassauwischen Wißbadischen Raht/ meinem günstigen Herrn.

44 Vgl. Held (ebd., fol. A2r-A2v): Es schreibt der Poet Sophocles in Aiace recht unnd wol/ da er also spricht: Decet virum memorem esse, si quid illi suave accidit. Gratia enim semper gratiam parit, cui vero accepti beneficii excidit memoria, is neutiquam est vir generosus. Das ist/ Es stehet einem Mann wol an/ daß er ingedenck seye/ so im etwas freundtlichs widerfert/ dann danck bringt danck/ so aber einer die empfangen gut und wolthaten in vergeß stellet/ der ist mit nichten für ein Bidermann zu halten. 
Literatur zugänglich ist, die er seinen Lesern über entsprechende Gedankengänge öffnet - was er im Weiteren denn auch noch mehrfach tut. Ganz ähnlich verfährt Held, wenn er lateinische Begriffe oder Redewendungen in seinen Text einflicht, die er mit einer deutschen Übersetzung oder Umschreibung verbindet. Nur den Terminus Emblemata lässt er als solchen stehen, den er offensichtlich als Gattungsbezeichnung auch denjenigen fassbar machen möchte, welche die Text-Bild-Konfigurationen bloß in den deutschen Übertragungen zur Kenntnis zu nehmen in der Lage sind. ${ }^{45}$ Im Kontext dieser Erwähnung wird deutlich, dass für Held exaktes philologisches Arbeiten nicht im Widerspruch zur kulturellen Transferleistung steht, wie er sie mit seinen Übersetzungen realisieren will. So konstatiert er:

derhalben [...] ich die Emblemata deß Hochgelehrten vnd Ehrvesten Herren Andree Alciati von Meiland/ der Rechten Doctor/ die ich in Teutsche Rheymen verfaßt vnd gebracht/ auch die Lateinischen Carmina vnd Verß auß vielen Exemplaren zusammen haltung vnd inspection treuwliche ersuchung der Griechischen Epigrammatum Planudis/ darauß dann die meisten vertiert vnd genommen worden seind/ emendiert und integritati restituiert habe. (fol. A4v-A5r) ${ }^{46}$

Wie Held hier versichert, ist er bei der Zusammenstellung der zu übertragenden Gedichte mit textkritischer Akribie vorgegangen, um die Epigramme für die Adressaten seiner zweisprachigen Ausgabe in einem Wortlaut zu bieten, der den humanistisch etablierten Maßstäben genügt. Auch wenn er die Beschreibung seiner philologischen Vorgehensweise auf die Ausgangstexte bezieht, verdeutlicht er seine Besorgnis um Textgenauigkeit letzten Endes ebenfalls hinsichtlich der eigenen Übersetzungen. Nicht nur durch die Zurschaustellung des methodischen Vokabulars, sondern vor allem auch über den expliziten Rückbezug auf die griechische Anthologie untermauert er den eigenen Sachverstand und dokumentiert, dass er genau weiß, wie und wo die Gedichtsammlung Alciatos zu verorten ist. Er ruft in Erinnerung, dass auch der Mailänder Jurist mit seinen Emblemen als Kulturvermittler fungiert, indem er die Planudea als Vorlage für viele der Epigramme wählte. In diesem Sinn sieht sich der deutsche Übersetzer mit dem eigenen Tun offensichtlich gleichsam als verlängerten Arm Alciatos.

45 Signifikanterweise benutzt Wolfgang Hunger im Kolumnentitel auf den Seiten mit den deutschen Epigrammen als Übersetzung des Plurals Emblemata die Formulierung verschroten werck: Damit ist genau bezeichnet, was das Wort emblema im Griechischen ursprünglich bedeutet, nämlich ,Schnitzwerk‘, ,Einlegearbeit‘.

46 Der Druck setzt lateinische Fachbegriffe in Antiqua, diese sind hier kursiv wiedergegeben. 
Indem Held bereits im Widmungsschreiben die meisten antiken Zitate und Fachwörter mit Übersetzungen ausstattet, holt er die Rezipienten dort ab, wo sie mit ihren Kenntnissen stehen. Auch die Wahl des Knittelverses für die Übertragungen der Subscriptiones spiegelt dieses Anliegen: Held fertigt die Übersetzungen nicht deswegen an, um neue Formen zu erproben oder zu zeigen, dass es möglich ist, derartige Gedichte auf deutsch zu gestalten, vielmehr möchte er Alciatos Embleme sowie das durch sie vermittelte Bildungsgut in den Erfahrungshorizont der Adressaten bringen. Dass es ihm nicht, wie Wolfgang Hunger, um den Stellenwert der deutschen Sprache geht, sondern sein Bestreben darin liegt, einer nicht im engeren Sinne humanistisch gebildeten Klientel Zugang zu einer Gattung zu verschaffen, die zu diesem Zeitpunkt gerade anfängt, europaweit für Aufsehen zu sorgen, wird auch in seiner Vorrede an den Günstigen Leser virulent, in welcher er die eigene Position nochmals schärfer konturiert.

In diesem zweiten Einleitungsstück rechtfertigt Held nicht nur, warum sich die Auseinandersetzung mit Alciatos Emblemen seiner Meinung nach besonders lohnt, sondern geht auch auf die Motive seines Übersetzens ein. Nachdem er in einer detaillierten Beweisführung mit dreizehn Argumentationspunkten dargelegt hat, welchen Nutzen die Beschäftigung mit den Text-Bild-Verbindungen bringt, fasst er den Zweck des Büchleins lapidar wie folgt zusammen:

in summa darauß man Lehr/ Vermanung/ Zucht/ Tugend/ gute Sitten und alle Bürgerliche ehrlichheit lehrnen und nemmen kan: darauß auch ein jeder sein Haußraht/ Gewand/ Teppich/ Schilt/ Wappen/ Helm/ Bitschier/ Wend/ Pfosten/ Küssin und ander ding mehr zeichen und zieren kan. (fol. B1v-B2r)

Held erkennt in Alciatos Emblembuch ein Lehrmittel für gute Sitten und bürgerliche Tugenden, rühmt es darüber hinaus gewissermaßen als lerntechnischen Multiplikator, indem er es als Lieferanten dekorativer Motive für allerlei Gegenstände des häuslichen Interieurs und des täglichen Gebrauchs anpreist. Über das skizzierte Anwendungsfeld umreißt der Übersetzer noch einmal die Zielgruppe seiner Übertragungen: Er richtet sich an eine gemischte Klientel von Adligen und wohlhabenden Bürgern, die es sich leisten kann, den bereits auf der Titelseite des Drucks genannten Berufsgruppen entsprechende Ausstattungsaufträge zu erteilen.

Dieses Bild bestätigt sich wenig später, als Held konkret auf seine Übersetzungstätigkeit zu sprechen kommt. So sei der Anstoß für die Übertragungen von außen gekommen, von etlich vom Adel vnd andere ehrliche Leut der Lateinischen Sprach vnerfahren (fol. B2r). Diese hätten Alciatos Büchlein bei ihm stehen sehen, sich einzelne Text-Bild-Konfigurationen erklären lassen und ihn - von den Emblemen fasziniert - gebeten, sie $z u$ verdeutschen vnd in gebreuchliche Reymen $z u$ 
bringen (ebd.). Held beschreibt sodann ausführlich sein Zögern, da er - abgesehen davon, dass er nie vil von dem Latein in das Teutsch vertiert (ebd.) habe - ein Problem darin zu erkennen glaubte, dass darinnen vil verborgner alter Historien/ Geschicht/ Fabeln und Geticht [...] waren/ die uns Teutschen unbekannt/ und mit so wenig worten nit verstanden hetten werden können (ebd.). Seine Bedenken zielten offensichtlich dahin, dass er Alciatos Emblembuch als zu voraussetzungsreich einschätzte, um es einem nicht humanistisch gebildeten Publikum ohne Weiteres näherzubringen.

Die ausgeführten Überlegungen spiegeln die Rolle, die sich Held hinsichtlich des Übersetzungsvorgangs schließlich zumaß: Er sah seine Aufgabe der Transposition darin, die Frucht seiner Tätigkeit dem Wissenshorizont der Adressaten anzupassen. Zugespitzt formuliert: Held erachtete im Grunde genommen einen doppelten Akt des Transfers für nötig, wobei der eine rein sprachlicher Natur ist, der zweite einen Adaptationsprozess mit Blick auf den Bildungsstand der intendierten Rezipienten impliziert.

Ganz anders als Hunger hat es Held nicht darauf angelegt, in einen Wettbewerb mit der Vorlage zu treten. Seine Übersetzungen beanspruchen keinen Eigenwert, sondern sollen dem Emblembuch den Zugang zu einer neuen Klientel eröffnen. Entsprechend äußert er sich zum Verhältnis zwischen Autor und Übersetzer:

allein habe ich disem Autori/ der vor Togam Latinam angehabt/ einen Teutschen Rock und Kleid angezogen/ daß er bey vns Teutschen auch vnder dem gemeinen Mann vngehindert wandern kündte vnd vilen nutz sey. (fol. B3v)

Auch diese Charakterisierung zielt auf eine Kultur der Vermittlung, ist Ausdruck der zweifachen Dolmetscherfunktion, die Held sich zuschreibt. Das Bild der UmGewandung korrespondiert mit der Vorstellung vom Übersetzer als jemandem, der nicht nur den sprachlichen Übertragungsakt leistet, sondern das Objekt seiner Bemühungen auch einem Assimilationsverfahren unterzieht.

Tatsächlich handelt es sich bei diesen programmatischen Überlegungen, wie Held sie hier festhält, nicht allein um Absichtserklärungen. Der Blick in die Epigramme zeigt, dass die in den Einleitungsstücken formulierten Grundsätze zweifelsohne die Leitlinien waren, denen der Übersetzer bei seiner Tätigkeit gefolgt ist. Weniger sprachliche Eleganz als Genauigkeit im Wortlaut prägt die übertragenen Epigramme, wobei Held offensichtlich immer dann von der Vorlage abwich, wenn er glaubte, inhaltliche Vereinfachungen vornehmen oder erklä- 
rende Zusatzinformationen ergänzen zu müssen, die das Verständnis des Sinns erleichtern. ${ }^{47}$

Zum Schluss seiner Vorrede an den Günstigen Leser pointiert Held noch einmal den hohen Stellenwert seiner Übersetzungsaufgabe. Er verdeutlicht den Rang des Autors seiner Vorlage, indem er auf andere Gelehrte verweist, die ebenfalls Gedichte aus der Anthologia Planudea ins Lateinische gebracht haben, so auf den Hoch und Wolgelährten Herrn Erasmus Roterodamus, Vincentius Obsopoeus, Philippus Melanthon, Thomas Venatorius, und andere (fol. B4r). Nicht nur, dass diese Aufzählung Andrea Alciato einem humanistisch wenig gebildeten Rezipientenkreis als jemanden vorstellt, der mit namhaften Persönlichkeiten wie Erasmus von Rotterdam oder Philipp Melanchthon in einem Atemzug genannt wird; mit der Anführung der Fachleute demonstriert Held auch erneut die eigene Sachkenntnis. Seinen Gipfel findet das Argument allerdings unmittelbar darauf in der Nennung Martin Luthers als deutschen Übersetzer der Fabeln Aesops, die dahin zielt, dass sich Held selbst mit seiner Tätigkeit an die Seite des prominenten Reformators stellt (fol. B4r).

Alles in allem sind Motivation und Zielrichtung der Alciato-Übersetzungen bei Held geprägt durch das Anliegen kulturdidaktischer Transgenese. Während Hunger bestrebt ist, mit seinen Übertragungen die deutsche Sprache zu nobilitieren und in ihrer Wertigkeit an die lateinische heranzurücken, versucht Held - in gewissem Sinne gerade konträr - die antik-gelehrten Inhalte an die volkssprachigen Verhältnisse anzupassen. Hunger betrachtet sich selbst als Dichter - er spricht von sich als Poeta Germanicus -, Held positioniert sich als Philologe und Vermittler, im Wesentlichen durch den Ehrgeiz getrieben, seinen Lesern eine unbekannte Welt zugänglich zu machen.

47 Für Beispiele siehe Daly: Introduction (Anm. 36), S. 15-20. 
Brought to you by | Universitaetsbibliothek Basel

Authenticated Download Date | 3/15/18 10:41 AM 